\title{
Algebro-Geometric Solutions for a Discrete Integrable Equation
}

\author{
Mengshuang Tao and Huanhe Dong \\ College of Mathematics and Systems Science, Shandong University of Science and Technology, Qingdao 266590, China
}

Correspondence should be addressed to Huanhe Dong; mathsdong@126.com

Received 25 April 2017; Revised 4 August 2017; Accepted 24 October 2017; Published 14 November 2017

Academic Editor: Chris Goodrich

Copyright (c) 2017 Mengshuang Tao and Huanhe Dong. This is an open access article distributed under the Creative Commons Attribution License, which permits unrestricted use, distribution, and reproduction in any medium, provided the original work is properly cited.

\begin{abstract}
With the assistance of a Lie algebra whose element is a matrix, we introduce a discrete spectral problem. By means of discrete zero curvature equation, we obtain a discrete integrable hierarchy. According to decomposition of the discrete systems, the new differential-difference integrable systems with two-potential functions are derived. By constructing the Abel-Jacobi coordinates to straighten the continuous and discrete flows, the Riemann theta functions are proposed. Based on the Riemann theta functions, the algebro-geometric solutions for the discrete integrable systems are obtained.
\end{abstract}

\section{Introduction}

As we all know, the generation of integrable system, determination of exact solution, and the properties of the conservation laws are becoming more and more rich [1-5]; in particular, the discrete integrable systems have many applications in statistical physics, quantum physics, and mathematical physics [6-11]. It is worth discussing the properties of discrete integrable systems, such as Darboux transformations [12, 13], Hamiltonian structures [14-16], exact solutions [17], and the transformed rational function method [18]. In the past decades, some methods have been proposed to gain explicit solutions of the continuous soliton equations, for instance, the algebro-geometric method $[19,20]$, the inverse scattering transformation [21], the Bäcklund transformation [22], and the sine-cosine method [23]. However, it is very hard to obtain algebro-geometric solutions for discrete soliton equations due to the treatment of discrete variables. In 1975, Its and Matveev first presented the algebro-geometric approaches [24], which permitted us to seek out a class of exact solutions to the soliton equations. The elliptic functions and multisoliton solutions may be acquired by these degenerated solutions [25]. Recently, Qiao et al. further improved the algebrogeometric methods by making use of the nonlinearization theory [26-29]. Trigonal curves are also systematically used to construct algebro-geometric solutions [30,31]. But we note that there is few research to focus on the algebro-geometric solutions of discrete soliton equations.
In this paper, we will generate the algebro-geometric solutions of the discrete integrable system by taking advantage of the Riemann-Jacobi inversion theorem and Abel coordinates. In Sections 2 and 3, we will construct a new discrete integrable system by using Lie algebra and spectral problem. By introducing Abel-Jacobi coordinates, straightening out of the continuous and discrete flows will be given and placed in Section 4. Section 5 will be devoted to derive the algebro-geometric solutions of the abovementioned discrete integrable equation by utilizing the Riemann theta function.

\section{The Discrete Integrable Hierarchy}

We consider the algebra

$$
\begin{aligned}
h_{1} & =\left(\begin{array}{ll}
1 & 0 \\
0 & 0
\end{array}\right), \\
h_{2} & =\left(\begin{array}{ll}
0 & 0 \\
0 & 1
\end{array}\right), \\
e & =\left(\begin{array}{ll}
0 & 1 \\
0 & 0
\end{array}\right), \\
f & =\left(\begin{array}{ll}
0 & 0 \\
1 & 0
\end{array}\right),
\end{aligned}
$$


which is the simple subalgebra of the Lie algebras $A_{1}$, and corresponding loop algebras can be expressed as

$$
\begin{gathered}
h_{i}(n)=h_{i} \lambda^{n}, \\
e(n)=e \lambda^{n}, \\
f(n)=f \lambda^{n},
\end{gathered}
$$

$$
n \in Z \text {. }
$$

According to the loop algebras, we introduce the following discrete spectral problems

$$
\begin{aligned}
& E \psi_{n}=U_{n} \psi_{n}, \\
& \frac{d \psi_{n}}{d t}=V_{n} \psi_{n},
\end{aligned}
$$

where

$$
\begin{aligned}
U_{n} & =h_{1}(-1)+h_{2}(-1)+r_{n} s_{n} h_{2}(1)+r_{n} e(1)+s_{n} f(1), \\
V_{n} & =\sum_{n=0}^{\infty}\left[a_{n}\left(h_{1}(-2 n)-h_{2}(-2 n)\right)+b_{n} e(-2 n+1)\right. \\
& \left.+c_{n} f(-2 n+1)\right] .
\end{aligned}
$$

Thus

$$
\begin{aligned}
U_{n} & =\left(\begin{array}{cc}
\frac{1}{\lambda} & r_{n} \\
s_{n} & \frac{1}{\lambda}+\lambda r_{n} s_{n}
\end{array}\right), \\
V_{n} & =\left(\begin{array}{cc}
A & B \\
C & -A
\end{array}\right),
\end{aligned}
$$

where

$$
\begin{aligned}
& A=\sum_{n=0}^{\infty} a_{n} \lambda^{-2 n}, \\
& B=\sum_{n=0}^{\infty} b_{n} \lambda^{-2 n+1}, \\
& C=\sum_{n=0}^{\infty} c_{n} \lambda^{-2 n+1} .
\end{aligned}
$$

According to the following stationary discrete zero curvature equation for $V_{n}$,

$$
\left(E V_{n}\right) U_{n}=U_{n} V_{n},
$$

we get

$$
\begin{aligned}
\frac{1}{\lambda}(E A-A)+s_{n} E B-r_{n} C & =0, \\
r_{n}(E A-A)+\left(\frac{1}{\lambda}+\lambda r_{n} s_{n}\right) E B-\frac{1}{\lambda} B & =0, \\
\frac{1}{\lambda} E C-s_{n}(E A+A)-\left(\frac{1}{\lambda}+\lambda r_{n} s_{n}\right) C & =0, \\
r_{n} E C-\left(\frac{1}{\lambda}+\lambda r_{n} s_{n}\right)(E A-A)-s_{n} B & =0 .
\end{aligned}
$$

Substituting (6) into (8) yields

$$
\begin{array}{r}
\Delta a_{n}+s_{n} E b_{n+1}-r_{n} c_{n+1}=0, \\
r_{n} s_{n} E b_{n+1}+r_{n}\left(E a_{n}+a_{n}\right)+\Delta b_{n}=0, \\
-s_{n} r_{n} c_{n+1}-s_{n}\left(E a_{n}+a_{n}\right)+\Delta c_{n}=0, \\
-r_{n} s_{n} \Delta a_{n+1}+r_{n} E c_{n+1}-s_{n} b_{n+1}-\Delta a_{n}=0,
\end{array}
$$

where $\Delta=E-1$.

We choose the initial values $a_{0}=-1 / 2, b_{0}=0$ and need to select zero constants for the inverse operation of the difference operator $\Delta$ in computing $a_{n}, n \geq 1$. On this condition, recursion relations (9) uniquely determine $a_{n}, b_{n}, c_{n}, n \geq 1$. Then, we obtain the first few quantities

$$
\begin{aligned}
& a_{1}=\frac{1}{s_{n-1} r_{n}}, \\
& b_{1}=\frac{1}{s_{n-1}}, \\
& c_{1}=\frac{1}{r_{n}}, \\
& a_{2}=-\frac{1}{s_{n-1}^{2} r_{n}{ }^{2}}-\frac{1}{r_{n}{ }^{2} s_{n} s_{n-1}}-\frac{1}{s_{n-1}^{2} r_{n-1} r_{n}}, \\
& b_{2}=-\frac{1}{s_{n-1}^{2} r_{n-1}}-\frac{1}{s_{n-1}^{2} r_{n}}, \\
& c_{2}=-\frac{1}{r_{n}^{2} s_{n-1}}-\frac{1}{r_{n}^{2} s_{n}}, \ldots
\end{aligned}
$$

From

$$
\begin{aligned}
& E \psi_{n}=U_{n} \psi_{n}, \\
& \frac{d \psi_{n}}{d t}=V_{n}^{(m)} \psi_{n},
\end{aligned}
$$

we have the discrete zero curvature equation

$$
\frac{d U_{n}}{d t_{m}}-\left(E V_{n}^{(m)}\right) U_{n}+U_{n} V_{n}^{(m)}=0,
$$

where

$$
V_{n}^{(m)}=\sum_{n=0}^{m}\left(\begin{array}{cc}
a_{n} \lambda^{2 m-2 n}-a_{m} & b_{n} \lambda^{2 m-2 n+1} \\
c_{n} \lambda^{2 m-2 n+1} & -a_{n} \lambda^{2 m-2 n}+a_{m}
\end{array}\right) .
$$


Thus, we obtain the following integrable discrete hierarchy

$$
\begin{aligned}
& r_{n, t_{m}}=E b_{m}-b_{m}, \\
& s_{n, t_{m}}=E c_{m}-c_{m} .
\end{aligned}
$$

And

$$
\begin{aligned}
& r_{n, t}=\frac{1}{s_{n}}-\frac{1}{s_{n-1}}, \\
& s_{n, t}=\frac{1}{r_{n+1}}-\frac{1}{r_{n}},
\end{aligned}
$$

with $m=1$. Equation (15) can be read as

$$
\begin{aligned}
& \partial_{t} \ln r_{n}=\frac{1}{r_{n}}\left(\frac{1}{s_{n}}-\frac{1}{s_{n-1}}\right), \\
& \partial_{\mathrm{t}} \ln s_{n}=\frac{1}{s_{n}}\left(\frac{1}{r_{n+1}}-\frac{1}{r_{n}}\right) .
\end{aligned}
$$

It is easy to find that the Lax pair of (15) is given by

$$
\begin{aligned}
E \varphi(n) & =U_{n} \varphi(n), \\
\varphi_{t}(n) & =V_{n}^{(1)} \varphi(n),
\end{aligned}
$$

where

$$
V_{n}^{(1)}=\left(\begin{array}{cc}
-\frac{1}{2} \lambda^{2} & \frac{1}{s_{n-1}} \lambda \\
\frac{1}{r_{n}} \lambda & \frac{1}{2} \lambda^{2}
\end{array}\right)
$$

In the following, we express Lenard's gradient sequences $S_{j}(0 \leq j \in Z)$, by the recursion equation

$$
\begin{aligned}
J_{n} S_{j+1}(n) & =K_{n} S_{j}(n), \\
J_{n} S_{-1}(n) & =0,
\end{aligned}
$$

$$
j=0,1, \ldots,
$$

with two operators

$$
\begin{gathered}
K_{n}=\left(\begin{array}{ccc}
\Delta & 0 & -s_{n}(E+1) \\
0 & \Delta & r_{n}(E+1) \\
r_{n} \Delta & s_{n} \Delta & -r_{n} s_{n} \Delta
\end{array}\right), \\
J_{n}=\left(\begin{array}{ccc}
r_{n} s_{n} & 0 & 0 \\
0 & -r_{n} s_{n} E & 0 \\
r_{n} \Delta & s_{n} \Delta & -r_{n} s_{n} \Delta
\end{array}\right),
\end{gathered}
$$

where $S_{j}(n)=\left(s_{j}^{(1)}, s_{j}^{(2)}, s_{j}^{(3)}\right)^{T}$.
From the equation $J_{n} S_{-1}(n)=0$ and (19), respectively,

$$
S_{-1}(n)=\left(\begin{array}{c}
0 \\
0 \\
-\frac{1}{2}
\end{array}\right) \text {, }
$$

$$
S_{0}(n)=\left(\begin{array}{c}
\frac{1}{r_{n}} \\
\frac{1}{s_{n-1}} \\
\frac{1}{s_{n-1} r_{n}}
\end{array}\right)
$$

$S_{1}(n)$

$$
=\left(\begin{array}{c}
-\frac{1}{r_{n}^{2}}\left(\frac{1}{s_{n-1}}+\frac{1}{s_{n}}\right) \\
-\frac{1}{s_{n-1}^{2}}\left(\frac{1}{r_{n-1}}+\frac{1}{r_{n}}\right) \\
-\frac{1}{s_{n-1} r_{n}}\left(\frac{1}{s_{n-1} r_{n}}+\frac{1}{s_{n} r_{n}}+\frac{1}{s_{n-1} r_{n-1}}\right)
\end{array}\right) .
$$

Equation (19) implies that

$$
\begin{array}{r}
\Delta s_{j}^{(2)}+r_{n}(E+1) s_{j}^{(3)}+r_{n} s_{n} E s_{j+1}^{(2)}=0, \\
\Delta s_{j}^{(1)}-s_{n}(E+1) s_{j}^{(3)}-r_{n} s_{n} s_{j+1}^{(1)}=0, \\
r_{n} \Delta s_{j}^{(1)}+s_{n} \Delta s_{j}^{(2)}-r_{n} s_{n} \Delta s_{j}^{(3)}=0 .
\end{array}
$$

The discrete integrable hierarchical (14) could be rewritten as generation of the following, so spectrum problem is

$$
\begin{aligned}
\psi(n+1) & =U_{n} \psi(n), \\
\psi(n)_{t_{m}} & =V_{n}^{(m)} \psi(n),
\end{aligned}
$$

where

$$
\begin{aligned}
& V_{n}^{(m)}=\left(\begin{array}{cc}
A_{n}^{(m)} & B_{n}^{(m)} \\
C_{n}^{(m)} & -A_{n}^{(m)}
\end{array}\right), \\
& A_{n}^{(m)}=\sum_{j=0}^{m} s_{j-1}^{(3)} \lambda^{2(m-j)}-s_{m}^{(3)}, \\
& B_{n}^{(m)}=\sum_{j=0}^{m} s_{j-1}^{(2)} \lambda^{2(m-j)+1}, \\
& C_{n}^{(m)}=\sum_{j=0}^{m} s_{j-1}^{(1)} \lambda^{2(m-j)+1} .
\end{aligned}
$$

From the compatibility conditions of the discrete Lax pair (23), we can read that the hierarchical equation is

$$
\begin{aligned}
& r_{n, t_{m}}=E s_{m-1}^{(2)}-s_{m-1}^{(2)}, \\
& s_{n, t_{m}}=E s_{m-1}^{(1)}-s_{m-1}^{(1)} .
\end{aligned}
$$


Thus, we also have

$$
\left(\begin{array}{c}
r_{n} \\
s_{n}
\end{array}\right)_{t_{m}}=X_{m}(n)=\left(\begin{array}{c}
\Delta s_{m-1}^{(2)} \\
\Delta s_{m-1}^{(1)}
\end{array}\right) .
$$

\section{Decomposition of the Differential-Difference Equations}

In this section, we shall resolve the discrete systems (16) into solvable ordinary differential equations. We assume that (23) has two basic solutions $\psi(n)=\left(\psi^{(1)}(n), \psi^{(1)}(n)\right)^{T}, \phi(n)=$ $\left(\phi^{(1)}(n), \phi^{(1)}(n)\right)^{T}$, and we define a Lax matrix $W_{n}$ as follows:

$$
\begin{aligned}
W_{n} & =\frac{1}{2}\left(\phi(n) \psi(n)^{T}+\psi(n) \phi(n)^{T}\right) \sigma \\
& =\left(\begin{array}{cc}
f(n) & g(n) \\
h(n) & -f(n)
\end{array}\right), \\
\sigma & =\left(\begin{array}{cc}
0 & -1 \\
1 & 0
\end{array}\right),
\end{aligned}
$$

and $W_{n}$ should meet the following equations:

$$
\begin{aligned}
W_{n+1} U_{n}-U_{n} W_{n} & =0, \\
W_{n, t_{m}} & =\left[V_{n}^{(m)}, W_{n}\right] .
\end{aligned}
$$

It is easy to see that (28) can be written as

$$
\begin{aligned}
& \frac{1}{\lambda} \Delta f(n)+s_{n} E g(n)-r_{n} h(n)=0, \\
& r_{n} E f(n)+\frac{1}{\lambda} \Delta g(n)+\lambda r_{n} s_{n} E g(n)+r_{n} f(n)=0, \\
& \frac{1}{\lambda} \Delta h(n)-s_{n} E f(n)-s_{n} f(n)-\lambda r_{n} s_{n} h(n)=0, \\
& r_{n} E h(n)-\frac{1}{\lambda} \Delta f(n)-\lambda r_{n} s_{n} \Delta f(n)-s_{n} g(n)=0, \\
& f(n)_{t_{m}}=B_{n}^{(m)} h(n)-B_{n}^{(m)} g(n), \\
& g(n)_{t_{m}}=2 A_{n}^{(m)} g(n)-2 B_{n}^{(m)} f(n), \\
& h(n)_{t_{m}}=2 C_{n}^{(m)} f(n)-2 A_{n}^{(m)} h(n),
\end{aligned}
$$

where

$$
\begin{aligned}
& f(n)=\sum_{j=0}^{N+1} f_{j-1}(n) \lambda^{2(N+1-j)}, \\
& g(n)=\sum_{j=1}^{N+1} g_{j-1}(n) \lambda^{2(N+1-j)+1}, \\
& h(n)=\sum_{j=1}^{N+1} h_{j-1}(n) \lambda^{2(N+1-j)+1} .
\end{aligned}
$$

Substituting (30) into (29) yields

$$
\begin{aligned}
J_{n} G_{j+1} & =K_{n} G_{j}(n), \\
J_{n} G_{-1}(n) & =0, \\
K_{n} G_{N-1}(n) & =0,
\end{aligned}
$$

$$
j \geq 0,
$$

where $G_{j}(n)=\left(h_{j}(n), g_{j}(n), f_{j}(n)\right)^{T}$.

It is evident that

$$
G_{-1}=\alpha_{0} S_{-1}(n),
$$

where $\alpha_{0}$ is a constant.

Acting with $J_{n}^{-1} K_{n}$ and $K_{n}^{-1} J_{n}$, respectively, on (32) yields

$$
G_{0}(n)=\alpha_{0} S_{0}(n)+\alpha_{1} S_{-1}(n) .
$$

Thus

$$
G_{k}(n)=\alpha_{0} S_{k}(n)+\alpha_{1} S_{k-1}(n)+\cdots+\alpha_{k+1} S_{-1}(n),
$$

where $\alpha_{0}, \alpha_{1}, \ldots, \alpha_{k+1}$ are constants.

Substituting (34) into the $K_{n} G_{N-1}(n)=0$ gives the following discrete stationary equation:

$$
\alpha_{0} X_{N}(n)+\alpha_{1} X_{N-1}(n)+\cdots+\alpha_{N} X_{0}(n)=0 .
$$

According to (31), we have $\left(\alpha_{0}=1\right)$

$$
\begin{aligned}
& h_{0}(n)=\frac{1}{r_{n}} \\
& g_{0}(n)=\frac{1}{s_{n-1}} \\
& f_{0}(n)=\frac{1}{r_{n} s_{n-1}}-\frac{1}{2} \alpha_{1}, \\
& h_{1}(n)=-\frac{1}{r_{n}^{2}}\left(\frac{1}{s_{n-1}}+\frac{1}{s_{n}}\right)+\alpha_{1} \frac{1}{r_{n}} \\
& g_{1}(n)=-\frac{1}{s_{n-1}^{2}}\left(\frac{1}{r_{n-1}}+\frac{1}{r_{n}}\right)+\alpha_{1} \frac{1}{s_{n-1}} .
\end{aligned}
$$

Define the elliptic coordinates $\mu_{j}$ and $\nu_{j}$ by expressing $g(n)$ and $h(n)$ :

$$
\begin{aligned}
g(n) & =\frac{1}{s_{n-1}} \lambda \prod_{j=1}^{N}\left(\lambda^{2}-\mu_{j}(n)^{2}\right) \\
& \equiv \frac{1}{s_{n-1}} \lambda \prod_{j=1}^{N}\left(\widetilde{\lambda}-\widetilde{\mu_{j}(n)}\right), \\
h(n) & =\frac{1}{r_{n}} \lambda \prod_{j=1}^{N}\left(\lambda^{2}-v_{j}(n)^{2}\right) \equiv \frac{1}{r_{n}} \lambda \prod_{j=1}^{N}\left(\widetilde{\lambda}-\widetilde{v_{j}(n)}\right),
\end{aligned}
$$

where we denote $\lambda^{2}, \mu_{j}(n)^{2}$, and $v_{j}(n)^{2}$ with $\tilde{\lambda}, \widetilde{\mu_{j}(n)}$, and $\widetilde{v_{j}(n)}$, respectively. 
By comparing coefficients of the same power for $\lambda$, we get

$$
\begin{aligned}
& g_{1}(n)=-\frac{1}{s_{n-1}} \sum_{j=1}^{N} \widetilde{\mu_{j}}(n), \\
& h_{1}(n)=-\frac{1}{r_{n}} \sum_{j=1}^{N} \widetilde{v_{j}}(n), \\
& g_{2}(n)=\frac{1}{s_{n-1}} \sum_{i<j}^{N} \widetilde{\mu_{i}}(n) \widetilde{\mu_{j}}(n), \\
& h_{2}(n)=\frac{1}{r_{n}} \sum_{i<j}^{N} \widetilde{v_{i}}(n) \widetilde{v_{j}}(n) .
\end{aligned}
$$

Equation (38) can be rewritten as

$$
\begin{gathered}
\frac{1}{s_{n-1}}\left(\frac{1}{r_{n-1}}+\frac{1}{r_{n}}\right)-\alpha_{1}=\sum_{j=1}^{N} \widetilde{\mu_{j}}(n), \\
\frac{1}{r_{n}}\left(\frac{1}{s_{n-1}}+\frac{1}{s_{n}}\right)-\alpha_{1}=\sum_{j=1}^{N} \widetilde{\gamma_{j}}(n),
\end{gathered}
$$

by making use of (37).

Thus, (16) can be written as

$$
\begin{aligned}
& \partial_{t} \ln r_{n}=\sum_{j=1}^{N} \widetilde{\nu_{j}}(n)+\alpha_{1}, \\
& \partial_{t} \ln s_{n}=\sum_{j=1}^{N} \widetilde{\mu_{j}}(n+1)+\alpha_{1} .
\end{aligned}
$$

Consider the function det $W_{n}$ which is a $(4 N+2)$ th-order polynomial in $\lambda$ :

$$
\begin{aligned}
-\operatorname{det} W_{n} & =f^{2}(n)+g(n) h(n)=\frac{1}{4} \lambda^{2} \prod_{j=1}^{2 N}\left(\lambda^{2}-\lambda_{j}^{2}\right) \\
& =\frac{1}{4} \widetilde{\lambda} \prod_{j=1}^{2 N}\left(\widetilde{\lambda}-\widetilde{\lambda_{j}}\right)=\frac{1}{4} R(\widetilde{\lambda}) .
\end{aligned}
$$

Substituting (30) into (41) and comparing coefficients of the same powers of $\lambda$ read

$$
\alpha_{1}=-\frac{1}{2} \sum_{j=1}^{2 N} \widetilde{\lambda_{j}}
$$

and deduce that

$$
\begin{aligned}
\left.f(n)\right|_{\tilde{\lambda}=\widetilde{\mu}_{k}(n)} & =\frac{1}{2} \sqrt{R\left(\widetilde{\mu}_{k}(n)\right),} \\
\left.f(n)\right|_{\tilde{\lambda}_{=} \tilde{\nu}_{k}(n)} & =\frac{1}{2} \sqrt{R\left(\widetilde{v}_{k}(n)\right),} \\
g(n)_{t_{1}} & =2 A_{n}^{(1)} g(n)-2 B_{n}^{(1)} f(n), \\
h(n)_{t_{1}} & =2 C_{n}^{(1)} f(n)-2 A_{n}^{(1)} h(n),
\end{aligned}
$$

by taking $m=1$.
Hence, it follows that

$$
\begin{aligned}
& \left.g(n)_{t_{1}}\right|_{\tilde{\lambda}=\widetilde{\mu}_{k}(n)}=-\frac{1}{s_{n-1}} \mu_{k}(n) \sqrt{R\left(\widetilde{\mu}_{k}(n)\right)} \\
& =-\frac{1}{s_{n-1}} \mu_{k}(n)\left(\partial_{t_{1}} \widetilde{\mu}_{k}(n)\right) \prod_{i=1, i \neq k}^{N}\left(\widetilde{\mu_{k}}(n)-\widetilde{\mu}_{i}(n)\right), \\
& \left.h(n)_{t_{1}}\right|_{\tilde{\lambda}=\widetilde{v}_{k}(n)}=\frac{1}{r_{n}} v_{k}(n) \sqrt{R\left(\widetilde{\nu}_{k}(n)\right)} \\
& =\frac{1}{r_{n}} v_{k}(n)\left(\partial_{t_{1}} \widetilde{v}_{k}(n)\right) \prod_{i=1, i \neq k}^{N}\left(\widetilde{v}_{k}(n)-\widetilde{v}_{i}(n)\right) .
\end{aligned}
$$

Again from (37) and (44), we have

$$
\begin{aligned}
& \frac{\partial_{t_{1}} \widetilde{\mu}_{k}(n)}{\sqrt{R\left(\widetilde{\mu}_{k}(n)\right)}}=-\frac{1}{\prod_{i=1, i \neq k}^{N}\left(\widetilde{\mu}_{k}(n)-\widetilde{\mu}_{i}(n)\right)}, \\
& \frac{\partial_{t_{1}} \widetilde{v}_{k}(n)}{\sqrt{R\left(\widetilde{v}_{k}(n)\right)}}=\frac{1}{\prod_{i=1, i \neq k}^{N}\left(\widetilde{v}_{k}(n)-\widetilde{v}_{i}(n)\right)} .
\end{aligned}
$$

Similarly, when $m=2$

$$
\begin{aligned}
& g(n)_{t_{2}} \mid \tilde{\lambda}_{=} \widetilde{\mu}_{k}(n) \\
& =-\left[\frac{1}{s_{n-1}} \mu_{k}(n)^{3}-\frac{1}{s_{n-1}^{2}}\left(\frac{1}{r_{n-1}}+\frac{1}{r_{n}}\right) \mu_{k}(n)\right] \\
& \cdot \sqrt{R\left(\widetilde{\mu}_{k}(n)\right)}=-\frac{1}{s_{n-1}} \mu_{k}(n)\left(\partial_{t_{2}} \widetilde{\mu}_{k}(n)\right) \\
& \cdot \prod_{i=1, i \neq k}^{N}\left(\widetilde{\mu_{k}}(n)-\widetilde{\mu}_{i}(n)\right), \\
& h(n)_{t_{2}} \widetilde{\lambda}_{\tilde{\gamma}=\widetilde{v}_{k}(n)}=\left[\frac{1}{r_{n}} v_{k}(n)^{3}-\frac{1}{r_{n}^{2}}\left(\frac{1}{s_{n-1}}+\frac{1}{s_{n}}\right) v_{k}(n)\right] \\
& \cdot \sqrt{R\left(\widetilde{v}_{k}(n)\right)}=\frac{1}{r_{n}} v_{k}(n)\left(\partial_{t_{2}} \widetilde{v}_{k}(n)\right) \\
& \cdot \prod_{i=1, i \neq k}^{N}\left(\widetilde{v}_{k}(n)-\widetilde{v}_{i}(n)\right) .
\end{aligned}
$$

Thus

$$
\begin{aligned}
& \frac{\partial_{t_{2}} \widetilde{\mu}_{k}(n)}{\sqrt{R\left(\widetilde{\mu}_{k}(n)\right)}}=-\frac{\widetilde{\mu}_{k}(n)-\sum_{j=1}^{N} \widetilde{\mu}_{j}(n)-\alpha_{1}}{\prod_{i=1, i \neq k}^{N}\left(\widetilde{\mu}_{k}(n)-\widetilde{\mu}_{i}(n)\right)}, \\
& \frac{\partial_{t_{2}} \widetilde{v}_{k}(n)}{\sqrt{R\left(\widetilde{v}_{k}(n)\right)}}=\frac{\widetilde{v}_{k}(n)-\sum_{j=1}^{N} \widetilde{\nu}_{j}(n)-\alpha_{1}}{\prod_{i=1, i \neq k}^{N}\left(\widetilde{v}_{k}(n)-\widetilde{v}_{i}(n)\right)} .
\end{aligned}
$$




\section{Straightening out of the Continuous and Discrete Flows}

In order to acquire the algebro-geometric solutions of systems (16), we first introduce the Riemann surface $\Gamma$ of the hyperelliptic curve with genus $N$ :

$$
\Gamma: \xi^{2}=R(\tilde{\lambda}), \quad R(\tilde{\lambda})=\prod_{j=1}^{2 N}\left(\tilde{\lambda}-\tilde{\lambda}_{j}\right),
$$

which has two infinite points $\infty_{1}$ and $\infty_{2}$, not branch point of $\Gamma$. We fix a set of regular cycle paths: $a_{1}, \ldots, a_{N} ; b_{1}, \ldots, b_{N}$, which are independent and have the intersection numbers:

$$
\begin{aligned}
& a_{k} \circ a_{j}=b_{k} \circ b_{j}=0, \\
& a_{k} \circ b_{j}=\delta_{k j},
\end{aligned}
$$

$$
1 \leq k, j \leq N
$$

We choose the holomorphic differentials, on $\Gamma$

$$
\widetilde{\omega}_{l}=\frac{\lambda^{l-1} d \lambda}{\sqrt{R(\lambda)}}, \quad 1 \leq l \leq N,
$$

and define

$$
\begin{aligned}
& A_{k j}=\int_{a_{j}} \widetilde{\omega}_{i}, \\
& B_{k j}=\int_{b_{j}} \widetilde{\omega}_{i},
\end{aligned}
$$

where $A=\left(A_{i j}\right)_{N \times N}, B=\left(B_{i j}\right)_{N \times N}$.

Thus, we denote the matrices $C$ and $\tau$ by

$$
\begin{aligned}
C & =\left(c_{k j}\right)=A^{-1}, \\
\tau & =A^{-1} B
\end{aligned}
$$

and verify that $\tau$ is symmetric and has positive defined imaginary part.

By normalizing $\widetilde{\omega}_{j}$ into the new basis $\omega_{j}$,

$$
\omega_{j}=\sum_{l=1}^{N-1} c_{j l} \widetilde{\omega}_{l}, \quad j=1,2, \ldots, N,
$$

which meets

$$
\begin{aligned}
& \int_{a_{k}} \omega_{j}=\sum_{l=1}^{N} c_{j l} \int_{a_{k}} \widetilde{\omega}_{l}=\sum_{l=1}^{N} c_{j l} A_{l k}=\delta_{j k}, \\
& \int_{b_{k}} \omega_{j}=\sum_{l=1}^{N} c_{j l} \int_{b_{k}} \widetilde{\omega}_{l}=\sum_{l=1}^{N} c_{j l} B_{l k}=\tau_{j k} .
\end{aligned}
$$

The Abel map $\mathscr{A}(p)$ is introduced as

$$
\mathscr{A}(p)=\int_{p_{0}}^{p} \omega
$$

and the Able-Jacobi coordinates are defined as

$$
\begin{aligned}
& \rho^{(1)}(n)=\mathscr{A}\left(\sum_{k=1}^{N} p\left(\tilde{\mu}_{k}(n)\right)\right)=\sum_{k=1}^{N} \int_{p_{0}}^{p\left(\widetilde{\mu}_{k}(n)\right)} \omega, \\
& \rho^{(2)}(n)=\mathscr{A}\left(\sum_{k=1}^{N} p\left(\widetilde{\nu}_{k}(n)\right)\right)=\sum_{k=1}^{N} \int_{p_{0}}^{p\left(\widetilde{v}_{k}(n)\right)} \omega,
\end{aligned}
$$

where

$$
\begin{aligned}
& p\left(\tilde{\mu}_{k}\right)=\left(\tilde{\lambda}=\tilde{\mu}_{k}(n), \xi=\sqrt{R\left(\tilde{\mu}_{k}(n)\right)}\right) \in \Gamma, \\
& p\left(\widetilde{\nu}_{k}\right)=\left(\tilde{\lambda}=\tilde{\nu}_{k}(n), \xi=\sqrt{R\left(\tilde{\nu}_{k}(n)\right)}\right) \in \Gamma,
\end{aligned}
$$

and $p_{0}$ is a chosen base point on $\Gamma$.

The components of the Abel-Jacobi coordinates in (56) are

$$
\begin{aligned}
& \rho_{j}^{(1)}(n)=\sum_{k=1}^{N} \int_{p_{0}}^{p\left(\widetilde{\mu}_{k}\right)} \omega_{j}=\sum_{k=1}^{N} \sum_{l=1}^{N} \int_{\tilde{\lambda}\left(p_{0}\right)}^{\widetilde{\mu}_{k}(n)} c_{j l} \frac{\tilde{\lambda}^{l-1} d \tilde{\lambda}}{\sqrt{R(\tilde{\lambda})}}, \\
& \rho_{j}^{(2)}(n)=\sum_{k=1}^{N} \int_{p_{0}}^{p\left(\widetilde{\nu}_{k}\right)} \omega_{j}=\sum_{k=1}^{N} \sum_{l=1}^{N} \int_{\tilde{\lambda}\left(p_{0}\right)}^{\tilde{\nu}_{k}(n)} c_{j l} \frac{\tilde{\lambda}^{l-1} d \tilde{\lambda}}{\sqrt{R(\tilde{\lambda})}},
\end{aligned}
$$

where $\tilde{\lambda}\left(p_{0}\right)$ is the local coordinate of $p_{0}$.

We infer that

$$
\begin{aligned}
\partial_{t} \rho_{j}^{(1)}(n) & =\sum_{k=1}^{N} \sum_{l=1}^{N} c_{j l} \frac{\tilde{\mu}_{k}(n)^{l-1} \partial_{t} \tilde{\mu}_{k}(n)}{\sqrt{R\left(\widetilde{v}_{k}(n)\right)}} \\
& =-\sum_{k=1}^{N} \sum_{l=1}^{N} c_{j l} \frac{\tilde{\mu}_{k}(n)^{l-1}}{\prod_{i=1, i \neq k}^{N}\left(\widetilde{\mu}_{k}(n)-\tilde{\mu}_{i}(n)\right)} \\
& =-c_{j N} \equiv \Omega_{j}^{(1)}, \\
\partial_{t} \rho_{j}^{(2)}(n) & =\sum_{k=1}^{N} \sum_{l=1}^{N} c_{j l} \frac{\widetilde{v}_{k}(n)^{l-1} \partial_{t} \widetilde{v}_{k}(n)}{\sqrt{R\left(\widetilde{v}_{k}(n)\right)}} \\
& =\sum_{k=1}^{N} \sum_{l=1}^{N} c_{j l} \frac{\widetilde{v}_{k}(n)^{l-1}}{\prod_{i=1, i \neq k}^{N}\left(\widetilde{v}_{k}(n)-\widetilde{v}_{i}(n)\right)}=c_{j N} \\
& \equiv-\Omega_{j}^{(1)} .
\end{aligned}
$$


Similarly, we have

$$
\begin{aligned}
& \partial_{t_{2}} \rho_{j}^{(1)}(n) \\
& =-\sum_{l=1}^{N} \sum_{k=1}^{N} c_{j l} \frac{\widetilde{\mu}_{k}(n)^{l-1}\left(\widetilde{\mu}_{k}(n)-\sum_{j=1}^{N} \widetilde{\mu}_{j}(n)-\alpha_{1}\right)}{\prod_{i=1, i \neq k}^{N}\left(\widetilde{\mu}_{k}(n)-\widetilde{\mu}_{i}(n)\right)} \\
& \quad=\Omega_{j}^{(2)}, \\
& \partial_{t_{2}} \rho_{j}^{(2)}(n) \\
& \quad=\sum_{l=1}^{N} \sum_{k=1}^{N} c_{j l} \frac{\widetilde{v}_{k}(n)^{l-1}\left(\widetilde{v}_{k}(n)-\sum_{j=1}^{N} \widetilde{\nu}_{j}(n)-\alpha_{1}\right)}{\prod_{i=1, i \neq k}^{N}\left(\widetilde{v}_{k}(n)-\widetilde{v}_{i}(n)\right)} \\
& =-\Omega_{j}^{(2)} .
\end{aligned}
$$

Let the fundamental solution matrix of (3) be of the form

$$
\begin{aligned}
& Q_{n}=(\phi(n), \widehat{\phi}(n))=\left(\begin{array}{lll}
\phi^{(1)} & (n) & \widehat{\phi}^{(1)}(n) \\
\phi^{(2)}(n) & \widehat{\phi}^{(2)}(n)
\end{array}\right), \\
& Q_{0}=I \text {. }
\end{aligned}
$$

It is easy to obtain that

$$
Q_{n+1}=U_{n} U_{n-1} \cdots U_{0},
$$

from which we have

$$
\begin{gathered}
\phi^{(1)}(1)=\frac{1}{\lambda}, \\
\phi^{(2)}(1)=s_{0}, \\
\widehat{\phi}^{(1)}(1)=r_{0}, \\
\widehat{\phi}^{(2)}(1)=\frac{1}{\lambda}+\lambda r_{0} s_{0}, \\
\phi^{(1)}(2)=\frac{1}{\lambda^{2}}+r_{1} s_{0}, \\
\phi^{(2)}(2)=s_{1} \frac{1}{\lambda}+s_{0}\left(\frac{1}{\lambda}+\lambda r_{1} s_{1}\right), \\
\widehat{\phi}^{(1)}(2)=r_{0} \frac{1}{\lambda}+\left(\frac{1}{\lambda}+\lambda r_{0} s_{0}\right) r_{1}, \\
\widehat{\phi}^{(2)}(2)=s_{1} r_{0}+\left(\frac{1}{\lambda}+\lambda r_{1} s_{1}\right)\left(\frac{1}{\lambda}+\lambda r_{0} s_{0}\right),
\end{gathered}
$$

Suppose that $\delta$ is eigenvalue of the Lax matrix $W_{n}$ in the solution space of equation $\psi(n+1)=U_{n} \psi(n)$, which is invariant under the action of $W_{n}$ due to $\left(E W_{n}\right) U_{n}=U_{n} W_{n}$. The corresponding eigenfunction is $\psi(n)$ that can be called the Baker function which satisfies that

$$
\begin{aligned}
& \psi(n+1)=U_{n} \psi(n), \\
& W_{n} \psi(n)=\delta \psi(n) .
\end{aligned}
$$

It is easy to check that

$$
\operatorname{det}\left|\delta I-W_{n}\right|=\delta^{2}-f^{2}(n)-g(n) h(n)=0,
$$

which has two eigenvalues $\delta^{ \pm}= \pm \delta$, where

$$
\delta=\sqrt{f^{2}(n)-g(n) h(n)}=\frac{1}{2} \sqrt{R(\tilde{\lambda})} .
$$

The corresponding Baker function can be taken as

$$
\begin{aligned}
& \phi^{ \pm}(n)=\phi(n)+b^{ \pm} \widehat{\phi}(n), \\
& \widehat{\phi}^{ \pm}(n)=\widehat{\phi}(n)+c^{ \pm} \phi(n),
\end{aligned}
$$

where

$$
\begin{aligned}
b^{ \pm} & =\frac{ \pm \delta-f(0)}{g(0)} \\
\text { or } b^{ \pm} & =\frac{h(0)}{ \pm \delta+f(0)}, \\
c^{ \pm} & =\frac{ \pm \delta+f(0)}{h(0)} \\
\text { or } c^{ \pm} & =\frac{g(0)}{ \pm \delta-f(0)} .
\end{aligned}
$$

Let $p^{ \pm}(n, \lambda), q^{ \pm}(n, \lambda)$ be the components of the Baker functions $\phi^{ \pm}(n)$ and $\widehat{\phi}^{ \pm}(n)$, respectively. Actually, starting from

$$
\begin{aligned}
Q_{n+1} & =U_{n} Q_{n} \\
W_{n} Q_{n} & =W_{n} U_{n-1} Q_{n-1}=\cdots=U_{n-1} U_{n-2} \cdots U_{0} W_{0} Q_{0} \\
& =Q_{n} W_{0} Q_{0}=Q_{n} W_{0},
\end{aligned}
$$

we can infer that

$$
\begin{aligned}
& p^{+}(n, \lambda) p^{-}(n, \lambda)=\frac{s_{-1}}{s_{n-1}} \prod_{j=1}^{N} \frac{\tilde{\lambda}-\tilde{\mu}_{j}(n)}{\tilde{\lambda}-\tilde{\mu}_{j}(0)}, \\
& p^{+}(n, \lambda) p^{-}(n, \lambda)=\frac{r_{0}}{r_{n}} \prod_{j=1}^{N} \frac{\tilde{\lambda}-\widetilde{v}_{j}(n)}{\tilde{\lambda}-\widetilde{v}_{j}(0)} .
\end{aligned}
$$

Similarly, we have

$$
\begin{aligned}
& \Delta \rho^{(1)}(n)=\rho^{(1)}(n+1)-\rho^{(1)}(n)=\Omega^{(0)}(\bmod \mathscr{J}), \\
& \Delta \rho^{(2)}(n)=\rho^{(2)}(n+1)-\rho^{(2)}(n)=\Omega^{(0)}(\bmod \mathscr{J}),
\end{aligned}
$$

where $\Omega^{(0)}=\int_{\infty_{1}}^{\infty_{2}} \omega$.

\section{Algebro-Geometric Solutions}

The well-known Riemann theta function of $\Gamma$ is defined by

$$
\theta(\xi \mid \tau)=\sum_{z \in Z^{N}} \exp (\pi i\langle\tau z, z\rangle+2 \pi i\langle\xi, z\rangle),
$$

$$
\xi \in C^{N} \text {, }
$$

where $\xi=\left(\xi_{1}, \ldots, \xi_{N}\right)^{T},\langle\xi, z\rangle=\sum_{j=1}^{N} \xi_{j} z_{j}$ 
According to the Riemann theorem, there exists a constant $M^{(i)} \in C^{N}$ so that

(i) $F_{1}=\theta\left(\mathscr{A}(p)-\rho^{(1)}(n)-M^{(1)}\right)$ has exactly $N$ zeros at $\tilde{\lambda}=\tilde{\mu}_{1}(n), \ldots, \tilde{\mu}_{N}(n)$;

(ii) $F_{2}=\theta\left(\mathscr{A}(p)-\rho^{(2)}(n)-M^{(2)}\right)$ has exactly $N$ zeros at $\tilde{\lambda}=\widetilde{v}_{1}(n), \ldots, \widetilde{v}_{N}(n)$.

We have the inversion formula

$$
\begin{aligned}
& \sum_{j=1}^{N} \widetilde{\mu}_{j}(n)^{k}=I_{k}(\Gamma)-\sum_{s=1}^{2} \operatorname{Res}_{\tilde{\lambda}=\infty_{s}} \tilde{\lambda}^{k} d \ln F_{1}(\widetilde{\lambda}), \\
& \sum_{j=1}^{N} \widetilde{v}_{j}(n)^{k}=I_{k}(\Gamma)-\sum_{s=1}^{2} \operatorname{Res}_{\tilde{\lambda}=\infty_{s}} \tilde{\lambda}^{k} d \ln F_{2}(\tilde{\lambda}),
\end{aligned}
$$

with the constant $I_{k}(\Gamma)=\sum_{j=1}^{N} \int_{a_{j}} \tilde{\lambda}^{k} \omega_{j}$. Through a standard treatment, we arrive at

$$
\begin{aligned}
& \sum_{j=1}^{N} \widetilde{\mu}_{j}(n)^{k}=I_{k}(\Gamma)+\partial_{t} \ln \frac{\theta\left(\rho^{(1)}(n)+M^{(1)}+\pi^{(2)}\right)}{\theta\left(\rho^{(1)}(n)+M^{(1)}+\pi^{(1)}\right)}, \\
& \sum_{j=1}^{N} \widetilde{v}_{j}(n)^{k}=I_{k}(\Gamma)+\partial_{t} \ln \frac{\theta\left(\rho^{(2)}(n)+M^{(2)}+\pi^{(1)}\right)}{\theta\left(\rho^{(2)}(n)+M^{(2)}+\pi^{(2)}\right)},
\end{aligned}
$$

where $\pi^{(s)}=\int_{\infty}^{p_{0}} \omega$.

Substituting (74) into (40) yields

$$
\begin{aligned}
r_{n} & =\exp \left[-\ln \frac{\theta\left(\rho^{(2)}(n)+M^{(2)}+\pi^{(1)}\right)}{\theta\left(\rho^{(2)}(n)+M^{(2)}+\pi^{(2)}\right)}-\frac{1}{2} t \sum_{j=1}^{2 N} \widetilde{\lambda}_{j}\right. \\
& \left.+I_{1}(\Gamma) t\right], \\
s_{n} & =\exp \left[-\ln \frac{\theta\left(\rho^{(1)}(n+1)+M^{(1)}+\pi^{(2)}\right)}{\theta\left(\rho^{(1)}(n+1)+M^{(1)}+\pi^{(1)}\right)}\right. \\
& \left.-\frac{1}{2} t \sum_{j=1}^{2 N} \tilde{\lambda}_{j}+I_{1}(\Gamma) t\right] .
\end{aligned}
$$

Thus

$$
\begin{aligned}
r_{n} & =\exp \left[-\ln \frac{\theta\left(\Omega^{(0)} n-\Omega^{(1)} t+\widehat{\Upsilon}^{(1)}\right)}{\theta\left(\Omega^{(0)} n-\Omega^{(1)} t+\widehat{\Upsilon}^{(2)}\right)}-\frac{1}{2} t \sum_{j=1}^{2 N} \tilde{\lambda}_{j}\right. \\
& \left.+I_{1}(\Gamma) t\right], \\
s_{n} & =\exp \left[-\ln \frac{\theta\left(\Omega^{(0)}(n+1)+\Omega^{(1)} t+\Upsilon^{(2)}\right)}{\theta\left(\Omega^{(0)}(n+1)+\Omega^{(1)} t+\Upsilon^{(1)}\right)}\right. \\
& \left.-\frac{1}{2} t \sum_{j=1}^{2 N} \widetilde{\lambda}_{j}+I_{1}(\Gamma) t\right]
\end{aligned}
$$

where

$$
\begin{aligned}
& \Upsilon^{(1)}=\rho_{0}^{(1)}+M^{(1)}+\pi^{(1)}, \\
& \Upsilon^{(2)}=\rho_{0}^{(1)}+M^{(1)}+\pi^{(2)}, \\
& \widehat{\Upsilon}^{(1)}=\rho_{0}^{(2)}+M^{(2)}+\pi^{(1)}, \\
& \widehat{\Upsilon}^{(2)}=\rho_{0}^{(2)}+M^{(2)}+\pi^{(2)} .
\end{aligned}
$$

which is the algebro-geometric solution to (16).

Remark 1. We have concluded the algebro-geometric solutions of the discrete system (16). It is significance of a major work for investigating numerical solutions of the discrete integrable system (16) like the way presented in [32]. Comparing the numerical solutions and algebro-geometric solutions about the discrete integrable system, we can get lots of useful properties. These problems will be studied in the future.

\section{Conflicts of Interest}

The authors declare that there are no conflicts of interest regarding the publication of this paper.

\section{Acknowledgments}

This work was supported by National Natural Science Foundation of China (no. 61402265 and no. 11701334), Open Fund of the Key Laboratory of Ocean Circulation and Waves, Chinese Academy of Sciences (no. KLOCAW1401), and the SDUST Research Fund (2014TDJH102).

\section{References}

[1] X. B. Hu, "A powerful approach to generate new integrable systems," Journal of Physics A: Mathematical and General, vol. 27, no. 7, pp. 2497-2514, 1994.

[2] X. Li and Q. Zhao, "A new integrable symplectic map by the binary nonlinearization to the super AKNS system," Journal of Geometry and Physics, vol. 121, pp. 123-137, 2017.

[3] X.-Y. Li, Q.-L. Zhao, Y.-X. Li, and H.-H. Dong, "Binary bargmann symmetry constraint associated with $3 \times 3$ discrete matrix spectral problem," Journal of Nonlinear Sciences and Applications, vol. 8, no. 5, pp. 496-506, 2015.

[4] X.-Y. Li, Y.-X. Li, and H.-X. Yang, "Two families of Liouville integrable lattice equations," Applied Mathematics and Computation, vol. 217, no. 21, pp. 8671-8682, 2011.

[5] H. Dong, K. Zhao, H. Yang, and Y. Li, "Generalised $(2+1)$ dimensional super MKdV hierarchy for integrable systems in soliton theory," East Asian Journal on Applied Mathematics, vol. 5, no. 3, pp. 256-272, 2015.

[6] X. Wang, H. Dong, and Y. Li, "Some reductions from a Lax integrable system and their Hamiltonian structures," Applied Mathematics and Computation, vol.218, no. 20, pp. 10032-10039, 2012.

[7] H. Yang, B. Yin, Y. Shi, and Q. Wang, "Forced ILW-Burgers equation as a model for Rossby solitary waves generated by topography in finite depth fluids," Journal of Applied Mathematics, vol. 2012, Article ID 491343, 2012. 
[8] T. C. Xia, "Two new integrable couplings of the soliton hierarchies with self-consistent sources," Chinese Physics B, vol. 19, no. 10, Article ID 100303, 2010.

[9] Y. Zhang, H. Dong, X. Zhang, and H. Yang, "Rational solutions and lump solutions to the generalized $(3+1)$-dimensional Shallow Water-like equation," Computers \& Mathematics with Applications. An International Journal, vol. 73, no. 2, pp. 246252, 2017.

[10] H. Yang, Q. Zhao, B. Yin, and H. Dong, "A new integrodifferential equation for rossby solitary waves with topography effect in deep rotational fluids," Abstract and Applied Analysis, vol. 2013, Article ID 597807, 2013.

[11] L.-Y. Tang and J.-C. Fan, "A family of Liouville integrable lattice equations and its conservation laws," Applied Mathematics and Computation, vol. 217, no. 5, pp. 1907-1912, 2010.

[12] Q.-L. Zhao, X.-Y. Li, and F.-S. Liu, "Two integrable lattice hierarchies and their respective Darboux transformations," Applied Mathematics and Computation, vol. 219, no. 10, pp. 5693-5705, 2013.

[13] X.-X. Xu, "A deformed reduced semi-discrete Kaup-Newell equation, the related integrable family and Darboux transformation," Applied Mathematics and Computation, vol. 251, pp. 275-283, 2015.

[14] Q.-1. Zhao and X.-Y. Li, "A Bargmann system and the involutive solutions associated with a new 4-order lattice hierarchy," Analysis and Mathematical Physics, vol. 6, no. 3, pp. 237-254, 2016.

[15] X.-X. Xu, "An integrable coupling hierarchy of the mKdVintegrable systems, Its Hamiltonian structure and corresponding nonisospectral integrable hierarchy," Applied Mathematics and Computation, vol. 216, no. 1, pp. 344-353, 2010.

[16] H.-X. Yang, J. Du, X.-X. Xu, and J.-P. Cui, "Hamiltonian and super-Hamiltonian systems of a hierarchy of soliton equations," Applied Mathematics and Computation, vol. 217, no. 4, pp. 1497$1508,2010$.

[17] R. Zhou and Q. Jiang, "A Darboux transformation and an exact solution for the relativistic Toda lattice equation," Journal of Physics A: Mathematical and General, vol. 38, no. 35, pp. 77357742, 2005.

[18] W.-X. Ma and J.-H. Lee, "A transformed rational function method and exact solutions to the $3+1$ math Container Loading Mathjax dimensional Jimbo-Miwa equation," Chaos Solitons and Fractals, vol. 42, no. 3, pp. 1356-1363, 2009.

[19] Y. Zhang and X.-J. Yang, "Generation of discrete integrable systems and some algebro-geometric properties of related discrete lattice equations," Journal of Nonlinear Sciences and Applications. JNSA, vol. 9, no. 12, pp. 6126-6141, 2016.

[20] X. Geng and C. Cao, "Quasi-periodic solutions of the $2+$ 1 dimensional modified Korteweg-de Vries equation," Physics Letters A, vol. 261, no. 5-6, pp. 289-296, 1999.

[21] M. J. Ablowitz and H. Segur, Solitons and the Inverse Scattering Transform, SIAM, Philadelphia, Pa, USA, 1981.

[22] S. X. Tao and T. C. Xia, "Binary nonlinearization of the super Broer-Kaup-KUPershmidt hierarchy," Chinese Annals of Mathematics. Series A, vol. 33, no. 2, pp. 217-228, 2012.

[23] H. W. Yang, Z. H. Xu, D. Z. Yang, X. R. Feng, B. S. Yin, and H. H. Dong, "ZK-Burgers equation for three-dimensional Rossby solitary waves and its solutions as well as chirp effect," Advances in Difference Equations, vol. 2016, no. 1, article 167, 2016.

[24] A. R. Its and V. B. Matveev, "Hill's operator with finitely many gaps," Functional Analysis and its Applications, vol. 9, no. 1, pp. 65-66, 1975.
[25] S. P. Novikov, S. V. Manakov, L. P. Pitaevskii, and V. E. Zakharov, Theory of Solitons: The Inverse Scattering Method, Plenum Press, New York, NY, USA, 1984.

[26] Z. J. Qiao, "A hierarchy of nonlinear evolution equations and finite-dimensional involutive systems," Journal of Mathematical Physics, vol. 35, no. 6, pp. 2971-2977, 1994.

[27] R. Zhou, "The finite-band solution of the Jaulent-Miodek equation," Journal of Mathematical Physics, vol. 38, no. 5, pp. 2535-2546, 1997.

[28] X. Geng and H. H. Dai, "Nonlinearization of the Lax pairs for discrete Ablowitz-LADik hierarchy," Journal of Mathematical Analysis and Applications, vol. 327, no. 2, pp. 829-853, 2007.

[29] J. Zhu and X. Geng, "Algebro-geometric constructions of the $(2+1)$-dimensional differential-difference equation," Physics Letters A, vol. 368, no. 6, pp. 464-469, 2007.

[30] W.-X. Ma, "Trigonal curves and algebro-geometric solutions to soliton hierarchies I," Proceedings of the Royal Society A, vol. 473, Article ID 20170232, 2017.

[31] W.-X. Ma, “Trigonal curves and algebro-geometric solutions to soliton hierarchies II," Proceedings of the Royal Society A, vol. 473, Article ID 20170233, 2017.

[32] M. J. Ablowitz and J. F. Ladik, "Nonlinear differential-difference equations and Fourier analysis," Journal of Mathematical Physics, vol. 17, no. 6, pp. 1011-1018, 1976. 


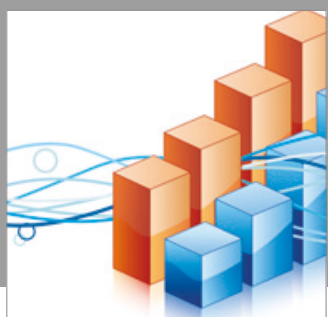

Advances in

Operations Research

vatersals

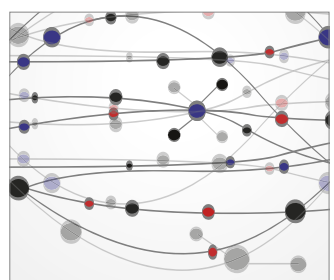

\section{The Scientific} World Journal
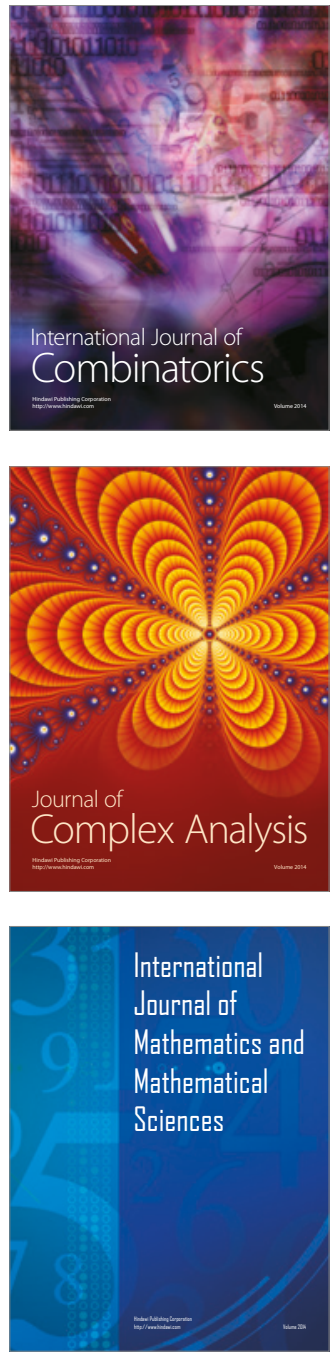
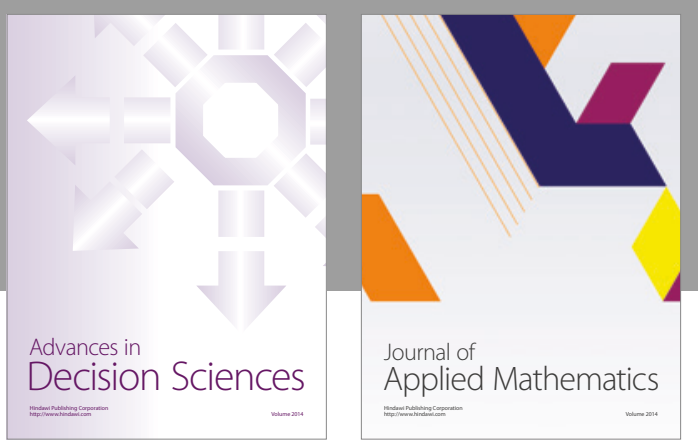

Algebra

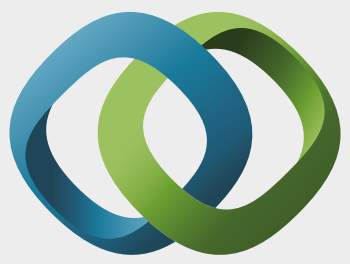

\section{Hindawi}

Submit your manuscripts at

https://www.hindawi.com
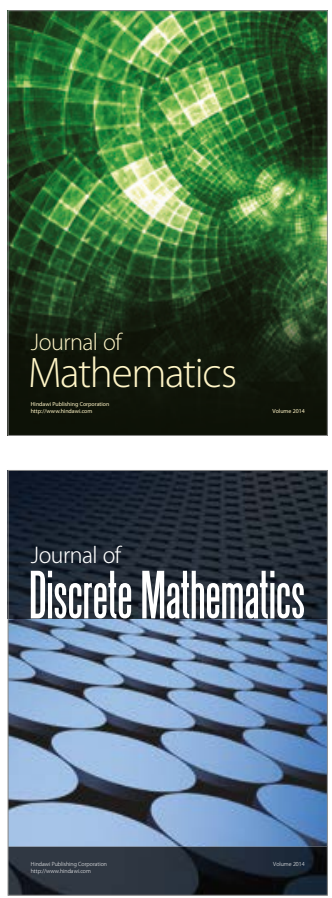

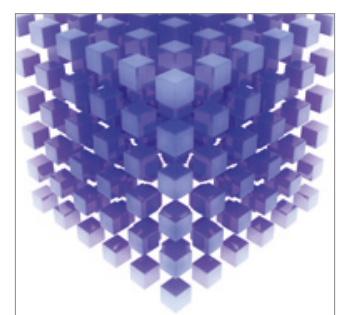

Mathematical Problems in Engineering
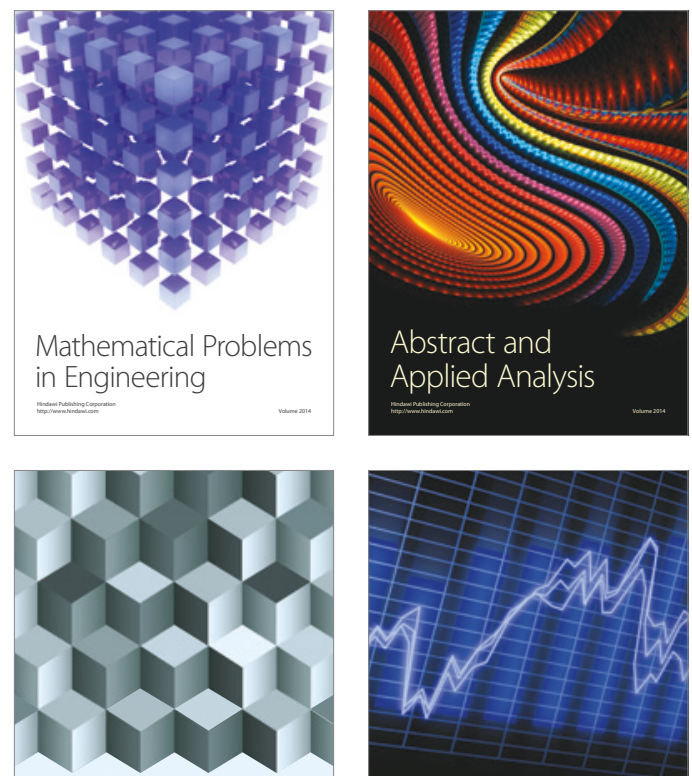

Journal of

Function Spaces

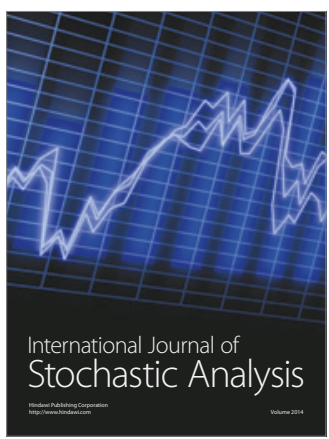

Probability and Statistics
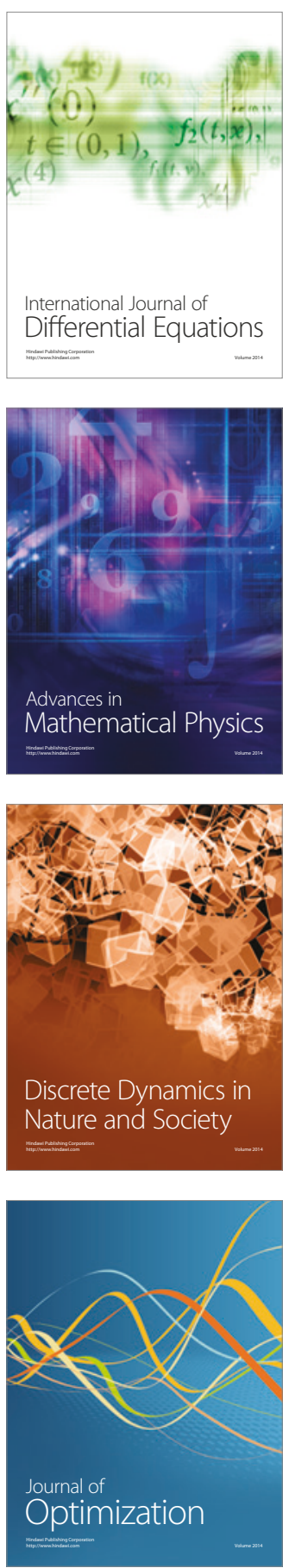